Arq. Bras. Med. Vet. Zootec., v.67, n.3, p.819-826, 2015

\title{
Parâmetros genéticos para características produtivas e biométricas em abelha Melipona quadrifasciata anthidioides LEPELETIER
}

[Genetic parameters for production and biometrics traits in honey bee Melipona quadrifasciata anthidioides LEPELETIER]

\author{
K.N. Oliveira' ${ }^{1}$, M.C. Paula-Leite ${ }^{2}$, P. Faquinello², C.A.L. Carvalho 2 , D.A. Lino-Lourenço ${ }^{3}$, \\ R.B. Sampaio ${ }^{1}$, E.B. Santos ${ }^{4}$ \\ ${ }^{1}$ Alunos pós-graduação - Universidade Federal do Recôncavo da Bahia - UFRB - Cruz das Almas, BA \\ ${ }^{2}$ Universidade Federal do Recôncavo da Bahia - UFRB - Cruz das Almas, BA \\ ${ }^{3}$ Universidade Estadual de Maringá - UEM - Maringá, PR \\ ${ }^{4}$ Aluno de graduação - Universidade Federal do Recôncavo da Bahia - UFRB - Cruz das Almas, BA
}

\section{RESUMO}

Os objetivos deste trabalho foram estimar componentes de variância genética aditiva, fenotípica e residual e a herdabilidade para características relacionadas com a produção de mel e com a estrutura do ninho de abelhas Melipona quadrifasciata anthidioides. Sessenta colônias de diferentes regiões da Bahia foram transladadas para caixas padronizadas modelo INPA e divididas, originando as gerações parentais G1 e G2. Foram medidas as características: estimativa da produção de mel; número, largura, volume e altura dos potes de mel; número, altura e diâmetro dos potes de pólen; peso; número, largura e diâmetro dos discos de cria e estimativa da população da colônia. As medidas foram corrigidas para o efeito fixo de mês de mensuração. Os componentes de variância e herdabilidade foram estimados por meio do método de semelhança entre parentes, utilizando-se

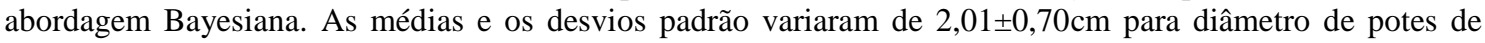
pólen a $2.333,0 \pm 384,1 \mathrm{~kg}$ para o peso das caixas. Houve indicação de convergência para todas as cadeias obtidas. As estimativas de variância genética aditiva variaram de $0,02 \mathrm{~cm}$ para as características largura dos potes de mel a $38.587,72 \mathrm{~kg}$ para o peso. Para as estimativas de variâncias fenotípicas, os valores variaram de 0,05 para a altura dos potes de pólen a $95.136,43 \mathrm{~kg}$ para o peso; e para as variâncias residuais, os valores encontrados variaram de 0,02 para a variável largura dos potes de mel a $56.548,71 \mathrm{~kg}$ para o peso. As estimativas de herdabilidade variaram de 0,35 a 0,53 . Os resultados demonstraram que as características avaliadas possuem variação genética aditiva que garante boa resposta à seleção.

Palavras-chave: avaliação genética, inferência Bayesiana, meliponicultura, seleção

\begin{abstract}
The aim of this study was to estimate components of genetic variance, phenotypic and residual and heritability for traits related to the production of honey and the nest structure of bee Melipona quadrifasciata anthidioides. Sixty colonies from different regions of Bahia were transferred to standard INPA model boxes and divided, creating the parental generations G1 and G2. The following characteristics were measured: estimated production of honey, number, width, height and volume of the honey pots, number, height and diameter of pollen storage pots, weight, number, length and diameter of the brood combs and estimate the population of the colony. The measurements were corrected for the fixed effects of month of measurement. The variance components and heritability were estimated by the method of similarity between relatives using the Bayesian approach. The mean and standard deviations ranged from $2.01 \pm 0.70 \mathrm{~cm}$ diameter pots for pollen to $2333.0 \pm 384.1 \mathrm{~kg}$ to the weight of the boxes. There was indication of convergence for all the chains obtained. Estimates of the additive genetic variance ranged from $0.02 \mathrm{~cm}$ to the width characteristics of honey pots to $38587.72 \mathrm{~kg}$ for weight. For the estimates of phenotypic variance the values ranged from 0.05 for the height of the pollen pots to $95136.43 \mathrm{~kg}$ for weight; and the residual variance the values varied from 0.02 for the variable width of the honey pots to $56548.71 \mathrm{~kg}$ for weight. The results showed that the characteristics assessed have additive genetic variation that ensures good response to selection.
\end{abstract}

Keywords: Bayesian inference, genetic evaluation, meliponiculture, selection

Recebido em 24 de abril de 2013

Aceito em 21 de agosto de 2014

E-mail: kalyoliveira@hotmail.com 


\section{INTRODUÇÃO}

No Brasil existe uma grande diversidade de espécies de abelhas sociais sem ferrão, com destaque para os meliponíneos, que possuem grande potencial para a produção de mel.

As abelhas do gênero Melipona quadrifasciata são encontradas no território brasileiro ao longo da costa leste, desde a Paraíba até o Rio Grande do Sul (Moure e Keer, 1950). Na Bahia, a espécie Melipona quadrifasciata anthidioides LEPELETIER possui ampla distribuição nas regiões secas. Possui mel de sabor apreciável e agradável, com alguns estudos envolvendo aspectos biológicos, morfológicos e genéticos (Aidar, 2010).

Os trabalhos realizados com essa espécie têm sido desenvolvidos para estimar parâmetros que possam ser usados na seleção de características produtivas da colônia, como o número total de potes de alimento, a altura, o diâmetro e o volume dos potes de mel e de pólen, e o número de discos de cria (Evangelista et al., 2008). Em abelhas do gênero Apis mellifera, alguns trabalhos têm sido realizados para estimar o comprimento da glossa de operárias (Souza et al., 2002) e o peso da rainha fisiogástrica (Barros, 2006).

Programas de melhoramento animal que envolvem a avaliação genética constituem-se em uma ferramenta fundamental nos sistemas de produção, por permitir a identificação dos melhores animais, resultando em um possível aumento da produtividade. Nesse sentido, é de grande importância a estimação acurada dos parâmetros e dos valores genéticos da população estudada.

Para proceder à avaliação genética de abelhas sem ferrão, não é possível a estimação dos componentes de variância utilizando-se os programas computacionais disponíveis para outras espécies de interesse zootécnico, como, por exemplo, bovinos, pois essas metodologias partem do princípio de que a esperança do parentesco entre irmãos completos é de 0,50; e entre tia e sobrinha é de 0,25 ; o que não é o caso das abelhas Melipona quadrifasciata anthidioides, que possuem comportamento monoândrico (Euán, 2005).
Dentre as metodologias de avaliação genética que podem ser empregadas no melhoramento animal, a análise Bayesiana apresenta-se como uma excelente alternativa para estimação dos componentes de (co)variância e parâmetros genéticos. Faria et al. (2007) citam que essa metodologia permite a obtenção de estimativas acuradas, apresentando maior flexibilidade, pelo fato de as distribuições marginais posteriores gerarem inferências mais precisas; e também que essa metodologia se destaca por permitir resolver problemas por meio de integrações numéricas, além de permitir o uso de uma pequena estrutura populacional, tornando o processo de avaliação mais preciso.

No gênero Apis mellifera, existem alguns trabalhos realizados que mostram valores de herdabilidades variando de 0,20 a 0,58 para característica de produção de mel, podendo haver mudança conforme o método de estimação e efeitos ambientais incluídos nos modelos estatísticos (Bienefeld e Pirchner, 1990). Entretanto, para a abelha Melipona quadrifasciata anthidioides LEPELETIER, as estimativas dos parâmetros genéticos para características produtivas e biométricas são inexistentes na literatura.

Objetivou-se neste trabalho estimar os componentes de variância genética aditiva, fenotípica e residual e a herdabilidade para as características relacionadas com a produção de mel e com a estrutura do ninho, que podem ser utilizadas em programas de seleção da espécie Melipona quadrifasciata anthidioides LEPELETIER.

\section{MATERIAL E MÉTODOS}

O trabalho foi desenvolvido no meliponário do Núcleo de Estudo dos Insetos - INSECTA, do Centro de Ciências Agrárias, Ambientais e Biológicas da Universidade Federal do Recôncavo da Bahia - UFRB, município de Cruz das Almas, Bahia $\left(12^{\circ} 3920 \mathrm{~W}\right.$ e $39^{\circ} 0723 \mathrm{~S}$, altitude $220 \mathrm{~m}$ ), durante o período de março de 2010 a julho de 2012.

Foram adquiridas sessenta colônias provenientes de diferentes cidades do Estado da Bahia, que, após um período de aclimatação de 30 dias, foram transladadas para caixas padronizadas modelo INPA, consistindo de fundo $(17 \times 17 \mathrm{~cm})$, 
ninho (medida interna $13 \times 13 \times 6,5 \mathrm{~cm}$ ), sobreninho (medida interna $13 \times 13 \times 6,5 \mathrm{~cm}$ ), melgueira (medida interna $13 \times 13 \times 5 \mathrm{~cm})$ e tampa $(17 \times 17 \mathrm{~cm})$. As colônias originais foram designadas de parentais e foram divididas após 90 dias, dando origem à geração G1, que foram novamente divididas após o mesmo período, originando a geração G2.

As divisões foram realizadas de acordo com o método de perturbação mínima (Oliveira e Kerr, 2000), utilizando um módulo tipo ninho vazio com um da colônia a ser dividida. Nas três gerações foram medidas as características peso da colônia (PESO); número, largura e diâmetro dos discos de cria (NDC, LDC, DDC, respectivamente); estimativa da produção de mel (PM); número, largura, volume e altura dos potes de mel (NPM, LPM, VPM, APM, respectivamente); número, altura e diâmetro dos potes de pólen (NPP, APP, DPP, respectivamente) e a estimativa da população (POP).

A variável PESO foi obtida pesando-se caixas vazias $(n=5)$ para a obtenção da média e subtraindo-se do peso da colônia, para se obter o peso líquido (discos de cria, cerume, potes com alimento, geoprópolis e abelhas), usando-se balança digital, seguindo-se metodologia utilizada por Alves (2010). As características LDC e DDC, APP e DPP, LPM e APM foram obtidas com o auxílio de uma régua graduada. $\mathrm{O}$ VPM foi mensurado por meio da sucção do conteúdo dos potes, com auxílio de seringas descartáveis graduadas.
A PM foi estimada por meio da multiplicação do número total de potes de mel e a média do volume dos potes de mel da colônia.

Para POP, primeiramente foi calculado o número de células de cria por meio da fórmula adaptada de Aidar (2010):

$N c=d m \times n f \times k$

Em que:

$N c=$ número de células de cria

$d m=$ diâmetro médio dos favos de cria

$n f=$ número de favos

$k=25$ constante do número de células por área (número de células / diâmetro do favo) para a espécie Melipona quadrifasciata anthidioides.

Assim a estimativa da POP foi obtida segundo a fórmula de Ihering (1932):

Pop $=n c+n c / 2$

Em que:

$n c=$ número de células de crias existentes na colônia.

Neste trabalho, para se proceder ao cálculo do grau de parentesco existente entre as abelhas Melipona quadrifasciata anthidioides (Fig. 1), foi utilizado o método da semelhança entre parentes, descrito por Falconer (1987), em que todas as operárias são filhas de mesmo pai, o relacionamento das operárias com as filhas de suas irmãs é de 0,375 e com as filhas da rainha é de 0,25 (Euán, 2005).

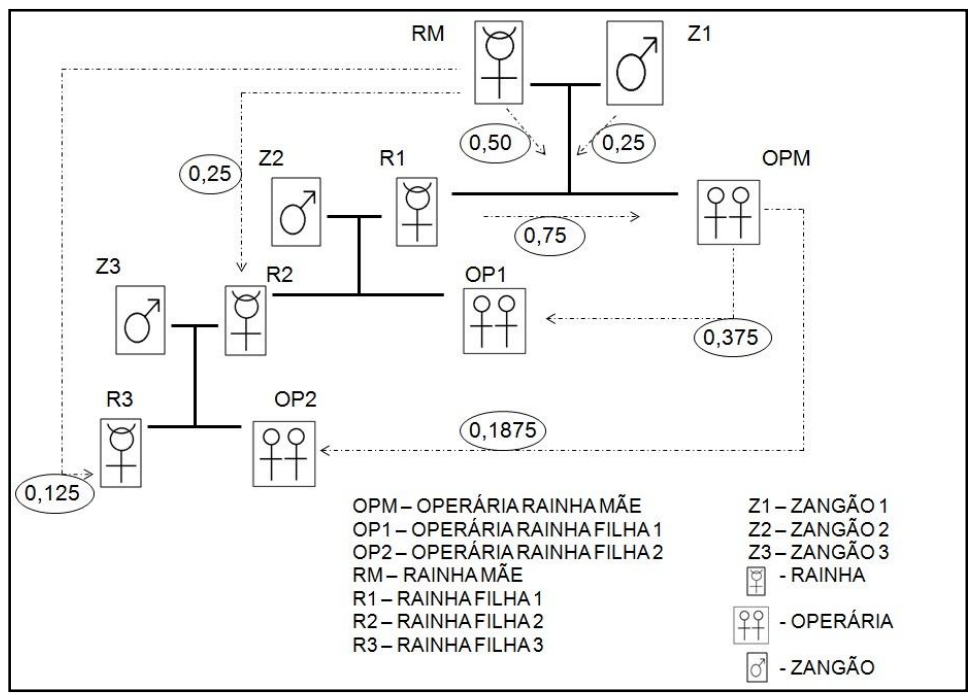

Figura 1. Relação de parentesco entre rainhas (RM, R1, R2 e R3) e operárias (OPM, OP1 e OP2) de abelhas Melipona quadrifasciata anthidioides, nas gerações Parental G1 e G2. 
Para a obtenção das estimativas dos componentes de variância e da herdabilidade, foi utilizada Inferência Bayesiana. Todas as medidas fenotípicas foram corrigidas para efeito fixo de mês de mensuração. Posteriormente, foi utilizado um modelo linear unicaracterística, e os algoritmos foram implementados por meio do software WinBUGS (Spiegelhalter et al., 2003). Os parâmetros genéticos foram estimados levando-se em consideração a média das características nas três gerações avaliadas.

Considerou-se que as observações possuíam distribuição normal multivariada, pelo fato de existir uma estrutura de correlação entre as três gerações, ou seja, ${ }_{i j} \sim N M V\left(\mu_{j}, V_{j}\right)$, em que: $y_{i j}$ é a observação $i$, tomada na geração $j$, e $V_{j}$ é a matriz de (co)variância fenotípica. Foi assumida distribuição de Wishart invertida para a matriz $V_{j}$, isto é, $V_{j} \sim I W\left(R_{j}, J\right)$, com $R_{j}=I_{j}=I_{3}$, em que $I$ é matriz identidade, e o parâmetro escala é igual a 3.

A variância genética foi calculada tomando-se como base que a (co)variância fenotípica entre parentes é igual ao coeficiente de parentesco multiplicado pela variância genética aditiva, adaptada para a seguinte forma:

$$
\sigma_{a_{k}}^{2}=\frac{n_{1} \operatorname{cov}\left(c_{i}, c_{i}+1\right) \frac{3}{8}+n_{2} \operatorname{cov}\left(c_{i}, c_{i}+1\right) \frac{3}{16}}{n_{1}+n_{2}}
$$

em que:

$\sigma_{a k}^{2}$ é a variância genética para a característica $k ; n_{l}$ é a soma do número de pares de observações entre tia e sobrinha, entre geração parental e G1, e entre G1 e G2; cov é a (co)variância; $c_{i}$ é a colônia da geração $i ; n_{2}$ é o número de pares de observações entre tia-avó e sobrinha-neta, ou seja, entre geração parental e $\mathrm{G} 2$. Os coeficientes $3 / 8$ e $3 / 16$ referem-se ao parentesco entre colônia tia e sobrinha, que é igual a 0,375 , e entre colônia tia-avó e sobrinhaneta, que é de 0,1875 .

Foi construída uma matriz de parentesco específica para o caso de abelhas sociais sem ferrão, que possuem comportamento monoândrico, e implementada a equação de modelos mistos, por meio do sistema computacional R (R Development Core Team,
2009), para a obtenção das estimativas de efeitos fixos para as rainhas e colônias das três gerações, em todas as características avaliadas.

Para cada componente de (co)variância, foram geradas 1.000 .000 de amostras em um processo MCMC (Monte Carlo Cadeias de Markov). A edição e análise de convergência das cadeias foram realizadas utilizando-se $\mathrm{o}$ sistema computacional R (R Development Core Team, 2009). Os testes de convergência aplicados foram o de Geweke (1992) e o de Heidelberger e Welch (1983).

\section{RESULTADOS E DISCUSSÃO}

$\mathrm{Na}$ Tabela 1, encontram-se as médias e os desvios padrão para as três gerações. Os valores obtidos variaram de $2,01 \pm 0,70 \mathrm{~cm}$ para diâmetro de potes de pólen (DPP) a 2.333,0 $0384,1 \mathrm{~kg}$ para o parâmetro peso (PESO).

Tabela 1. Médias e desvio padrão das características biométricas e produtivas em abelha Melipona quadrifasciata anthidioides LEPELETIER (Hymenoptera: Apidae)

\begin{tabular}{lcc}
\hline \multicolumn{1}{c}{ Características } & Média & Desvio Padrão \\
\hline NPM (un) & 29,98 & 14,77 \\
LPM (cm) & 2,10 & 0,14 \\
VPM (ml) & 5,73 & 1,17 \\
APM (cm) & 2,65 & 0,28 \\
NPP (un) & 3,04 & 3,45 \\
APP (cm) & 2,91 & 0,50 \\
DPP (cm) & 2,01 & 0,70 \\
PM (ml) & 175,06 & 99,78 \\
PESO (kg) & $2.333,00$ & 384,10 \\
NDC (un) & 5,10 & 1,33 \\
LDC (cm) & 5,55 & 0,91 \\
DDC (cm) & 5,81 & 0,95 \\
POP (un) & $1.116,67$ & 350,38 \\
\hline
\end{tabular}

(NPM), (LPM), (APM) e (VPM): número, largura, altura e volume dos potes de mel; (NPP), (APP) e (DPP): número, altura e diâmetro dos potes de pólen; (PM): produção de mel; (PESO): peso da colônia; (NDC), (DDC) e (LDC): número, diâmetro e largura dos discos de cria; (POP): número de indivíduos.

Os resultados são semelhantes aos encontrados por Darakjiian (1989) e Alves (2007), em trabalhos realizados com espécies do mesmo gênero, em que encontraram valores que variaram de 500 a 2.000 para número de indivíduos; $3,02 \pm 0,43 \mathrm{~cm}$ para altura de potes de pólen e $2,48 \pm 0,90 \mathrm{~cm}$ para diâmetro de potes de pólen, respectivamente. Os valores médios 
encontrados para NDC foram de 5,10 $\pm 1,33$ discos. Esse valor foi superior ao sugerido por Aidar (2010) como ideal para divisão da colônia, em que, no mínimo, deve apresentar 5 discos de cria, sendo que 3 devem ser pré-nascentes.

Os valores obtidos para a estimativa da PM, PESO, NPM e para VPM foram inferiores aos encontrados por Monteiro (2000), que cita que, em ambiente natural, essa espécie pode produzir de 1,5 a 2,0 litros de mel/colônia, em boa florada, e criada racionalmente, a produtividade pode aumentar. Barros (2006) realizou trabalhos de seleção da espécie Melipona scutellaris e encontrou valores de produção de mel que variaram de $4.280,0 \mathrm{~kg}$ a $7.980,0 \mathrm{~kg}$; Dias et al. (2008) encontraram valores médios de 55,43 potes de mel em colmeias de abelha Jandaíra (Melipona subnitida) alimentadas artificialmente; estudos com volume médio de potes de mel, envolvendo a espécie Melipona scutellaris, indicam um volume médio de 14,13ml por pote (Alves et al., 2005).

Houve indicação de convergência para todas as cadeias por meio da utilização dos testes de diagnósticos.

As estimativas de variância genética aditiva obtida variaram de 0,02 $\pm 0,01$ a 4.121,84 $\pm 2.374,69$, observando-se maior média para a característica PM e a menor, para LPM (Tab. 2). Para as estimativas de variâncias

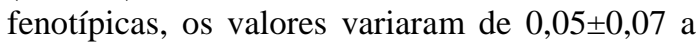
8.168,88 $\pm 1.170,83$; e, para as variâncias residuais, os valores encontrados variaram de 0,02 $\pm 0,01$ a 4.047,04 $\pm 2 \cdot 347,37$ (Tab. 2). Assim como nas estimativas de variância genética aditiva, o maior valor encontrado para variâncias fenotípicas e residuais foi para a característica PM e o menor, para a LPM, devido à magnitude dessas características.

Tabela 2. Estimativas de variância genética aditiva $\left(\sigma_{\mathrm{a}}^{2}\right)$, variância fenotípica $\left(\sigma_{\mathrm{p}}^{2}\right)$, residual $\left(\sigma_{\mathrm{e}}^{2}\right)$ e de herdabilidade $\left(\mathrm{h}^{2}\right)$, e seus respectivos desvios padrão (DP) e intervalos de credibilidade (entre parênteses), no nível de 95\%, para as características número (NPM), largura (LPM), volume (VPM) e altura dos potes de mel (PPM), número (NPP), altura (APP) e diâmetro dos potes de pólen (DPP) e produção de mel (PM), obtidas para abelhas Melipona quadrifasciata anthidioides LEPELETIER (Hymenoptera: Apidae)

\begin{tabular}{|c|c|c|c|c|c|c|c|c|}
\hline \multirow[t]{2}{*}{ Características } & \multicolumn{2}{|c|}{$\left(\sigma_{\mathrm{a}}^{2}\right)$} & \multicolumn{2}{|c|}{$\sigma_{p}^{2}$} & \multicolumn{2}{|c|}{$\left(\sigma_{\mathrm{e}}^{2}\right)$} & \multicolumn{2}{|l|}{$\left(h^{2}\right)$} \\
\hline & Média & $\mathrm{DP}$ & Média & $\mathrm{DP}$ & Média & $\mathrm{DP}$ & Média & DP \\
\hline NPM & $\begin{array}{c}94,15 \\
(6,52-191,80)\end{array}$ & 51,61 & $\begin{array}{c}178,43 \\
(135,20- \\
236,60)\end{array}$ & 25,97 & $\begin{array}{c}84,29 \\
(4,60-186,77)\end{array}$ & 51,44 & $\begin{array}{c}0,53 \\
(0,03-0,97)\end{array}$ & 0,27 \\
\hline LPM & $\begin{array}{c}0,02 \\
(0,0007-0,04)\end{array}$ & 0,01 & $\begin{array}{c}0,05 \\
(0,03-0,06)\end{array}$ & 0,07 & $\begin{array}{c}0,02 \\
(0,002-0,05)\end{array}$ & 0,01 & $\begin{array}{c}0,40 \\
(0,02-0,93)\end{array}$ & 0,26 \\
\hline VPM & $\begin{array}{c}0,51 \\
(0,02-1,25)\end{array}$ & 0,35 & $\begin{array}{c}1,23 \\
(0,93-1,61)\end{array}$ & 0,17 & $\begin{array}{c}0,72 \\
(0,06-1,33)\end{array}$ & 0,34 & $\begin{array}{c}0,41 \\
(0,01-0,94)\end{array}$ & 0,27 \\
\hline APM & $\begin{array}{c}0,04 \\
(0,001-0,10)\end{array}$ & 0,03 & $\begin{array}{c}0,11 \\
(0,08-0,14)\end{array}$ & 0,02 & $\begin{array}{c}0,07 \\
(0,01-0,12)\end{array}$ & 0,03 & $\begin{array}{c}0,35 \\
(0,01-0,90)\end{array}$ & 0,25 \\
\hline NPP & $\begin{array}{c}1,86 \\
(0,06-5,00)\end{array}$ & 1,38 & $\begin{array}{c}5,12 \\
(3,90-6,76)\end{array}$ & 0,73 & $\begin{array}{c}3,26 \\
(0,43-5,67)\end{array}$ & 1,38 & $\begin{array}{c}0,36 \\
(0,01-0,91)\end{array}$ & 0,25 \\
\hline APP & $\begin{array}{c}0,05 \\
(0,003-0,11)\end{array}$ & 0,03 & $\begin{array}{c}0,09 \\
(0,07-0,13)\end{array}$ & 0,02 & $\begin{array}{c}0,05 \\
(0,003-0,10)\end{array}$ & 0,03 & $\begin{array}{c}0,51 \\
(0,03-0,97)\end{array}$ & 0,28 \\
\hline DPP & $\begin{array}{c}0,04 \\
(0,001-0,10)\end{array}$ & 0,03 & $\begin{array}{c}0,09 \\
(0,07-0,14)\end{array}$ & 0,02 & $\begin{array}{c}0,06 \\
(0,006-0,11)\end{array}$ & 0,03 & $\begin{array}{c}0,39 \\
(0,01-0,94)\end{array}$ & 0,27 \\
\hline PM & $\begin{array}{c}4121,84 \\
(253,80- \\
8.707,00) \\
\end{array}$ & $2.374,69$ & $\begin{array}{c}8.168,88 \\
(6.214,00- \\
10.790,00)\end{array}$ & $1.170,83$ & $\begin{array}{c}4.047,04 \\
(241,00- \\
8.593,00)\end{array}$ & $2.347,37$ & $\begin{array}{c}0,50 \\
(0,03-0,97)\end{array}$ & 0,27 \\
\hline
\end{tabular}

Com relação às estimativas de herdabilidade $\left(\mathrm{h}^{2}\right)$, as mesmas variaram de 0,35 a 0,53 para as características produtivas (Tab. 2) e de 0,40 a 0,53 para as características biométricas (Tab. 3), indicando que essas características podem ser selecionadas e incluídas em um processo de seleção. 
$\mathrm{Na}$ Tabela 3, encontram-se as estimativas dos parâmetros genéticos e fenotípicos para as características biométricas.

Os valores encontrados para variância genética aditiva $\left(\sigma_{\mathrm{a}}^{2}\right.$ ) variaram de 0,29 a $38.587,72$, sendo o maior valor observado para a variável PESO e o menor, para DDC (Tab. 3). Para os parâmetros de variância fenotípica, os valores variaram de $0,65 \pm 0,09$ a $95.136,43 \pm 14.005,45$; e para a variância residual, os valores obtidos variaram de

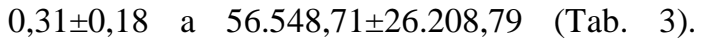
Observou-se a maior estimativa para a característica PESO e a menor, para a LDC, tanto para variância fenotípica como para a residual, por causa da magnitude das características.

Tabela 3. Estimativas dos componentes de variância genética aditiva $\left(\sigma_{\mathrm{a}}^{2}\right)$, fenotípica $\left(\sigma_{\mathrm{p}}^{2}\right)\left(\sigma_{\mathrm{p}}^{2}\right)$ e residual $\left(\sigma_{\mathrm{e}}^{2}\right)$, e de herdabilidade $\left(\mathrm{h}^{2}\right)$, com seus respectivos intervalos de credibilidade (IC) e desvios padrão (DP) para as características peso da colônia (PESO), número (NDC), largura (LDC) e diâmetro dos discos de cria (DDC) e estimativa da população (POP), obtidas para abelhas Melipona quadrifasciata anthidioides LEPELETIER (Hymenoptera: Apidae)

\begin{tabular}{ccccc}
\hline Características & Parâmetros & Média & DP & IC \\
& $\left(\sigma_{\mathrm{a}}^{2}\right)$ & $38.587,72$ & $26.511,65$ & $(1.694,00-96.080,00)$ \\
PESO & $\left(\sigma_{\mathrm{p}}^{2}\right)$ & $95.136,43$ & $14.005,45$ & $(72.000,0-126.800,0)$ \\
& $\left(\sigma_{\mathrm{e}}^{2}\right)$ & $56.548,71$ & $26.208,79$ & $(6.070,0-104.040,0)$ \\
& $\left(\mathrm{h}^{2}\right)$ & 0,40 & 0,26 & $(0,01-0,93)$ \\
\hline \multirow{2}{*}{ NDC } & $\left(\sigma_{\mathrm{a}}^{2}\right)$ & 0,39 & 0,25 & $(0,02-0,91)$ \\
& $\left(\sigma_{\mathrm{p}}^{2}\right)$ & 0,89 & 0,12 & $(0,68-1,16)$ \\
& $\left(\sigma_{\mathrm{e}}^{2}\right)$ & 0,50 & 0,25 & $(0,04-0,95)$ \\
& $\left(\mathrm{h}^{2}\right)$ & 0,43 & 0,27 & $(0,02-0,95)$ \\
\hline \multirow{2}{*}{ LDC } & $\left(\sigma_{\mathrm{a}}^{2}\right)$ & 0,35 & 0,19 & $(0,02-0,70)$ \\
& $\left(\sigma_{\mathrm{p}}^{2}\right)$ & 0,65 & 0,09 & $(0,49-0,85)$ \\
& $\left(\sigma_{\mathrm{e}}^{2}\right)$ & 0,31 & 0,18 & $(0,02-0,67)$ \\
& $\left(\mathrm{h}^{2}\right)$ & $0,53-0,97)$ \\
\hline \multirow{2}{*}{ DDC } & $\left(\sigma_{\mathrm{a}}^{2}\right)$ & 0,29 & 0,27 & $(0,01-0,68)$ \\
& $\left(\sigma_{\mathrm{p}}^{2}\right)$ & 0,67 & 0,19 & $(0,51-0,87)$ \\
& $\left(\sigma_{\mathrm{e}}^{2}\right)$ & 0,38 & 0,09 & $(0,03-0,71)$ \\
& $\left(\mathrm{h}^{2}\right)$ & 0,43 & 0,18 & $(0,02-0,94)$ \\
\hline & $\left(\sigma_{\mathrm{a}}^{2}\right)$ & $23.238,84$ & $16.458,63$ & $(957,39-59.550,00)$ \\
& $\left(\sigma_{\mathrm{p}}^{2}\right)$ & $59.758,86$ & $8.265,17$ & $(45.800,0-78.180,0)$ \\
& $\left(\sigma_{\mathrm{e}}^{2}\right)$ & $36.520,02$ & $16.224,41$ & $(4.240,0-65.146,0)$ \\
& $\left(\mathrm{h}^{2}\right)$ & 0,38 & 0,26 & \\
\hline
\end{tabular}

Neste estudo, as estimativas de variância genética aditiva $\left(\sigma_{\mathrm{a}}^{2}\right)$, variância fenotípica $\left(\sigma_{\mathrm{p}}^{2}\right)$, variância residual $\left(\sigma_{\mathrm{e}}^{2}\right)$ e herdabilidade $\left(\mathrm{h}^{2}\right)$ para todas as características foram obtidas com precisão. De maneira geral, observa-se maior valor para a variância fenotípica $\left(\sigma_{\mathrm{p}}^{2}\right)$, indicando que a mesma pode ser responsável pela variação existente nas características avaliadas.
Os resultados obtidos neste trabalho, com a utilização de análise unicaracter, foram satisfatórios, com valores de herdabilidades de magnitude moderada a alta; entretanto, estudos subsequentes devem ser realizados com o objetivo de obter a correlação genética entre essas características. 


\section{CONCLUSÕES}

As estimativas dos componentes de variância e de herdabilidade revelaram que as características avaliadas podem ser utilizadas como critério de seleção em programas de melhoramento da espécie de abelha Melipona quadrifasciata anthidioides LEPELETIER.

\section{AGRADECIMENTOS}

À FAPESB, pelo recurso financeiro referente aos termos de outorga PPP0064/2010, BOL1836/2010 e BOL0529/2011, e ao CNPq, pelas bolsas referentes aos processos 552415/2010-3 e 303237/2010-4.

\section{REFERÊNCIAS}

AIDAR, D.S. A mandaçaia: biologia de abelhas, manejo e multiplicação artificial de colônias de Melipona quadrifasciata Lep. (Hymenoptera, Apidae, Meliponinae). Ribeirão Preto: FUNPECEditora, 2010. 161p.

ALVES, R.M.O. Avaliação de parâmetros biométricos e produtivos para seleção de

ALVES, R.M.O.; SOUZA, B.A.; CARVALHO, C.A.L. Ninhos de Melipona scutellaris em coqueiros na região do Litoral Norte e Metropolitana do Estado da Bahia. Mensagem Doce, v.1, p.10-13, 2005.

ALVES, R.M.O.; SOUZA, B.A.; CARVALHO, C.A.L. Notas Sobre a Bionomia de Melipona mandacaia (Apidae : Meliponini). Magistra, v.19, p.177-264, 2007.

colônias da Abelha Uruçu (Melipona scutellaris Latreille, 1811). 2010. 104f. Tese (Doutorado em Ciências Agrárias) - Universidade Federal do Recôncavo da Bahia, Brasil.

BARROS, J. de R.S. Genetic breeding on the bee Melipona scutellaris (Apidae, Meliponinae). Rev. Acta Amazônica. v.36, p.115-120, 2006.

BIENEFELD, K.; PIRCHNER, F. Heritabilities for several colony traits in the honeybee (Apis mellifera carnica). Apidologie, v.21, p.175-183, 1990.

DARAKJIIAN, P. Biologia geral da mandaçaia. Apicultura e Polinização, v.31, p.24-25, 1989.
DIAS, V.H.P.; FILGUEIRA, M.A.; OLIVEIRA, F.L.; DIAS, A.M. Alimentação artificial à base de mel e suas implicações no desenvolvimento de famílias de abelhas jandaíras (Melipona subnitida Ducke) em Mossoró - RN. Rev. Verde de Agroecol. e Desenvol. Sustentável. v.1, p.4044, 2008.

EVANGELISTA-RODRIGUES, A.; GÓIS, G.C.; SILVA, C.M. Desenvolvimento produtivo de colmeias de abelhas Melipona scutellaris. Biotemas, v.21, p.59-64, 2008.

EUÁN, J.J.G.Q. Biología y uso de las abejas sin aguijón de la península de Yucatán, Mexico (Hymenoptera: Meliponini). Mérida: Universidad Autónoma de Yucatán, 2005. 115p.

FALCONER, D.S. Introdução a genética quantitativa. Viçosa: UFV, 1987. 279p.

FARIA, C.U.; MAGNABOSCO, C.U.; REYES, A.L. et al. Inferência Bayesiana e sua Aplicação na Avaliação Genética de Bovinos da Raça Nelore: Revisão Bibliográfica. Ciênc. Anim. Bras., v.8, p.75-86, 2007.

GEWEKE, J.; BERNARDO, J.M.; BERGER, J. et al. Evaluating the accuracy of sampling-based approaches to the calculation of posterior moments (with discussion). In: Bayesian statistics, 4.ed. Oxford: Oxford University Press, 1992. p.169-193.

HEIDELBERGER, P.; WELCH, P. Simulation run length control in the presence of an initial transient. Operations Rese., v.31, p.1109-1144, 1983.

IHERING, H.A. Uruçu na Apicultura Nordestina. Chácaras e Quintais. v.46, p.292296, 1932.

MONTEIRO, W.R. Meliponicultura (Criação de abelhas sem ferrão). Mensagem Doce, v.57, p.1517, 2000.

MOURE, J.S.; KERR, W.E. Sugestões para modificação da sistemática do gênero Melipona (Hymenoptera, Apoidea). Dusenia, v.18, p.10529, 1950.

OLIVEIRA F.; KERR, W.E. Divisão de uma colônia de jupará (Melipona compressipes manaosensis) usando-se uma colmeia e o método de Fernando Oliveira. INPA, v.10, 2000. 7p. 
R DEVELOPMENT CORE TEAM. $R: A$ language and environment for statistical computing. $\mathrm{R}$ Foundation for Statistical Computing, Vienna, Austria. ISBN 3-90005107-0, URL http://www.R-project.org. 2009.

SOUZA, D.C.; CRUZ, C.D.; CAMPOS, L.A. Correlation between honey production and some morphological traits in Africanized honey bees (Apis mellifera). Ciencia Rural. v.32, p.869-872, 2002.
SPIEGELHALTER, D.; THOMAS, A.; BEST, N.; LUNN, D. WinBUGS User Manual: Version 1.4. Cambridge: MRC Bioestatistics Unit, 2003. 